\title{
Assessment and comparison of target registration accuracy in surgical instrument tracking technologies
}

\author{
Teatini A. ${ }^{1,2}$, Pérez de Frutos J. ${ }^{3,4}$, Lang $\varnothing$ T. ${ }^{3}$, Edwin B. ${ }^{1,5}$, Elle O. ${ }^{1,2}$ Member, IEEE
}

\begin{abstract}
Image guided surgery systems aim to support surgeons by providing reliable pre-operative and intra-operative imaging of the patient combined with the corresponding tracked instrument location. The image guidance is based on a combination of medical images, such as Magnetic Resonance Imaging (MRI), Computed Tomography (CT) and Ultrasonography (US), and surgical instrument tracking. For this reason, tracking systems are of great importance as they determine location and orientation of the equipment used by surgeons. Assessment of the accuracy of these tracking systems is hence of the utmost importance to determine how much error is introduced in image guided surgery only due to tracking inaccuracy. Thus, this study aimed to compare in a surgical Operating Room (OR) accuracy of the two most used tracking systems, Optical Tracking (OT) and Electromagnetic Tracking (EMT), in terms of Target Registration Error (TRE) assessment at multiple distances from the target position. Results of the experiments show that optical tracking performs more accurately in tracking the instrument tip than electromagnetic tracking in the experimental conditions. This was tested using a phantom designed for accuracy measurement in a wide variety of positions and orientations. Nevertheless, EMT remains a viable option for flexible instruments, due to its reliability in tracking without the need for line of sight.
\end{abstract}

\section{INTRODUCTION}

In Image Guided Surgery (IGS), instrument tracking technologies are of paramount importance as they provide reliable information regarding position and orientation of surgical instruments and are also used to navigate the surgeon throughout the procedure. These technologies have been commonly used in neurosurgical applications [1]. However, in the past decade, application of IGS has expanded also to other surgical procedures, such as endoscopic sinus surgery [2] and laparoscopic nephrectomy [3]. Moreover, research is currently progressing to introduce image guidance in more complex procedures, such as laparoscopic liver resection or pancreatic ablation and resection [4], [5].

This paper is part of the research in image guided systems for laparoscopic liver resection surgery, as part of the H2020-

*Study supported by H2020-MSCA-ITN Marie Skłodowska-Curie Actions, Innovative Training Networks (ITN) - H2020 MSCA ITN 2016 GA EU project number 722068 High Performance Soft Tissue Navigation (HiPerNav).
MSCA-ITN EU project number 722068, High Performance Soft Tissue navigation (HiPerNav*). The liver represents one of the most frequent targets of metastases from other cancers, such as colorectal cancers, for which the estimated number of cases in a year is 550000 patients worldwide [6]. An IGS based on pre-operative CT or MRI scans would aid the surgeon towards the target metastases and spare as much healthy tissue as possible around the tumor. The most commonly used tracking systems for navigation in clinical research are OT and EMT systems. OT makes use of triangulation of light rays either reflected by a set of markers, or generated by infrared emitting diodes, to provide location and orientation of the tracked tool. However, in case of occlusion of the optical markers, i.e. loss of line of sight between cameras and markers, tracking information is interrupted. Instead, EMT makes use of magnetic fields to find fluxgate sensors or search coils, thus without relying on line of sight for tracking. This feature of EMT permits tracking of sensors inside the abdomen of the patient. Moreover, due to the small dimensions of the sensors, it is possible to position the sensor very close to the tool tip, and hence also track flexible instruments. Nonetheless, EMT sensors present a relatively small measurement volume with comparison to OT [7]. The EMT measurement volume can also be distorted and even reduced due to ferromagnetic interference of metallic objects close to the EMT field generator [8].

The purpose of this study is to assess and compare the accuracy of both OT and EMT in terms of Target Registration Error (TRE). TRE is a measure of accuracy in tracking a position on a tool at a certain distance from the sensors. For a surgical instrument, this equates to tracking the tip of the tool. TRE is defined [9] as follows:

$$
\operatorname{TRE}^{2}(\mathrm{x})=\frac{\mathrm{FLE}^{2}}{\mathrm{~N}}\left(1+\frac{1}{3} \sum_{\mathrm{k}=1}^{3} \frac{\mathrm{d}_{\mathrm{k}}^{2}}{\mathrm{f}_{\mathrm{k}}^{2}}\right)
$$

where $x$ is an arbitrary target point, $N$ the number of markers, $d_{k}$ the distance $x$ from each axis $k, f_{k}$ the RMS of the distance and FLE stands for Fiducial Localization Error, which is a

\footnotetext{
${ }^{1}$ The Intervention Center, Oslo University Hospital, Oslo, Norway

${ }^{2}$ Department of Informatics, University of Oslo, Oslo, Norway

${ }^{3}$ Department of Health research, SINTEF AS, Trondheim, Norway

${ }^{4}$ Department of Computer Science, NTNU, Trondheim, Norway

${ }^{5}$ Oslo University Hospital, Department of Hepato-Pancreatic-Biliary surgery, Oslo, Norway
} 
distance between a marker and its measured position [10]. TRE can be measured using a theoretical approach through (1) or via direct measurement using a phantom [11]. For this study, a direct measurement approach using a phantom was preferred over the theoretical approach to provide results closer to those of a realistic surgical scenario. Moreover, the experiment was carried out in an OR on a conventional OR table used for laparoscopic surgery in St. Olav's hospital, Trondheim.

\section{MAterials AND MethodS}

\section{A. Phantom Design}

ASTM standard F2554-10 for optical tracking accuracy measurement [12] was used as basis to design the phantom. The phantom design was improved to enable analysis of TRE also for electromagnetically tracked instruments. The phantom was built in polyoxymethylene thermoplastic (because of its hardness and low deformability), which prevents magnetic interference to EMT. Moreover, the phantom was designed to cover positions within a volume of interest (operating volume) approximately as in laparoscopic liver resection procedures. Hence, its dimensions are: $150 \mathrm{~mm}$ height, $200 \mathrm{~mm}$ width and $300 \mathrm{~mm}$ depth. A total of 71 target positions were machined onto the phantom, divided into 28 titanium target divot pins for TRE and 43 vertically drilled target holes. The titanium pins present a conical shape at 1 $\mathrm{mm}$ depth and $60^{\circ}$ angle. The divot holes are designed to be complementary to a pointer adapter. The 43 vertical drilled holes are $2 \mathrm{~mm}$ in diameter and $10 \mathrm{~mm}$ deep, designed to hold an Northern Digital Inc. ${ }^{\circledR}$ (NDI) [13] EMT catheter sensor probe based on previous experiences from other studies [8], [14] but were not used throughout this study. The target positions, according to standard ASTM F2554-10, are placed at five different orientations, over a total of seven different planes.

Optical and electromagnetic reference sensors were rigidly connected through a bracket to the phantom to track the position of the phantom on the surgical table (NDI® 6DOF Reference Disk Part Number 610066 and Polaris Rigid Body Part Number 8700339). The reference support bracket allows for stable and repeatable repositioning of the reference sensors. The aforementioned pointer adapter was manufactured to allow testing of differently sized surgical instruments. This is also built in polyoxymethylene. It is designed as a cylindrical plastic holder with threaded holes located $120^{\circ}$ apart. These are meant for tightening screws. A titanium tip, $1 \mathrm{~mm}$ in diameter and $3 \mathrm{~mm}$ in length, is found at the opposite side of the adapter, which fits perfectly in the titanium target pins of the phantom, as mentioned before. The phantom and tool adapter can be seen in Fig. 1. A MICROCUT® 837-I milling machine, equipped with Mitutoyo ${ }^{\circledR}$ AT115 Linear Scales and a metal tip to reach inside the divots were used to accurately measure the positions of the target divots on the phantom. The system comprises two $600 \mathrm{~mm}$ linear scales for displacements on the horizontal plane, and one $1100 \mathrm{~mm}$ linear scale on the vertical axis. The measuring accuracy is $8 \mu \mathrm{m}$ for horizontal displacements and of $10 \mu \mathrm{m}$ for vertical movements [15].

\section{B. Experimental Protocol}

The phantom was placed on a MAQUET® surgical table in the OR (Fig. 1). Both tracking systems were positioned in the configuration they will have during navigation in laparoscopic abdominal surgery, according to discussions with the surgeons. A Planar Field NDI Aurora ${ }^{\circledR}$ V2 system electromagnetic generator was rigidly connected to the surgical table using the field generator mounting arm of NDI® on the assistant side of the surgical table i.e., left patient supine side. This position of the EMT generator avoids occlusion line of sight of the interventional staff in visualizing anesthesia data and would only represent an insignificant burden to the assisting surgeon [8]. The optical tracking system NDI Polaris Spectra ${ }^{\circledR}$ was positioned at the head of the surgical table. The Phantom was positioned on the right side of the surgical table to replicate the position of the liver of the patient. The positions of the tracking systems were slightly adjusted in order to allow the measurement volumes of both tracking systems to maintain instrument tracking throughout all target positions of the phantom.

Accuracy of the tracking systems in terms of TRE was evaluated through two experiments. Experiment 1 examined the accuracy when the sensors, for EMT NDI 6DOF flex Tube $1.3 \mathrm{~mm}{ }^{\circledR}$ Part Number 610060 (accuracy specifications for NDI ${ }^{\circledR}$ sensors can be found in reference material provided by NDI®) and for OT a custom built optical marker frame with NDI ${ }^{\circledR}$ passive infrared markers, were positioned on a rigid wooden instrument at four distances from the tool tip, classified as: A, B, C and D with increasing distances from the tool tip, as visible in Fig. 1. The positions of the sensors were extracted using the recordings from the NDI Toolbox ${ }^{\circledR}$ software. The tool tip was calibrated from the sensor positions through the pivoting functionality provided in the software. This reflects a real-life scenario in which tools are sterilized and calibrated through pivoting in the OR once clamped to the surgical instrument. The tracked tool tip was then shifted into

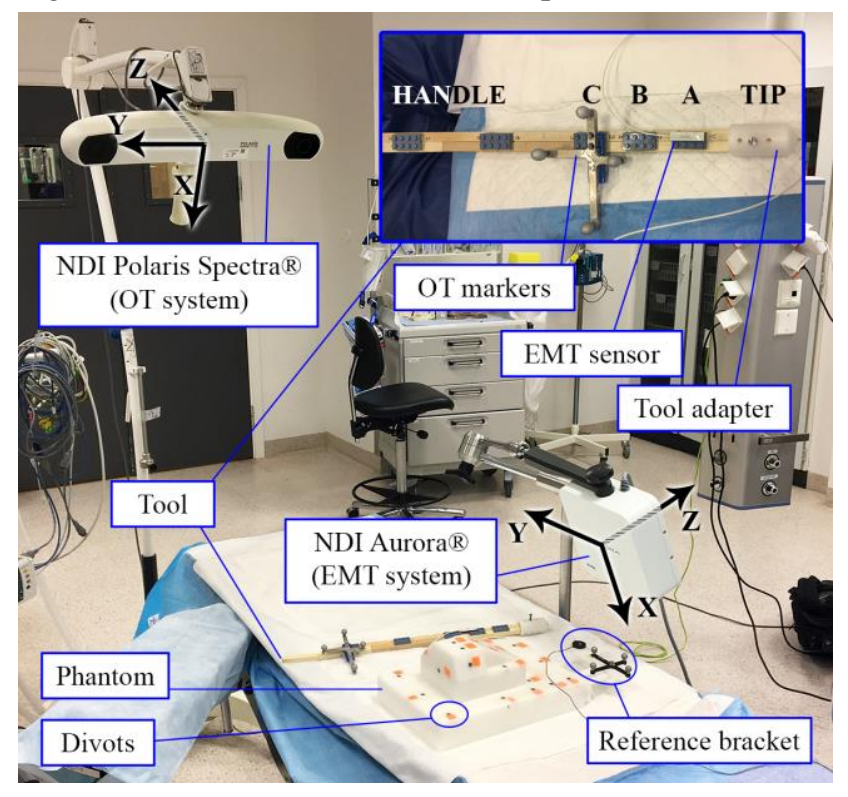

Fig. 1 Experimental Setup, with optical and electromagnetic tracking systems facing the TRE phantom. 
Table 1. Results of the study, errors in $x, y, z$ axes are with respects to the axes of the Polaris cameras and Aurora generator, visible in Fig. 1.

\begin{tabular}{|c|c|c|c|c|c|c|c|c|}
\hline \multirow{2}{*}{$\begin{array}{c}\text { Tracking } \\
\text { System }\end{array}$} & \multicolumn{8}{|c|}{ Accuracy (mm) } \\
\hline & Position & Pivot (RMS) & $\begin{array}{c}\text { Pivot Error } \\
\text { (RMS) }\end{array}$ & Error $x$ & Errory & Error $z$ & $M e a n \pm S D$ & Max error \\
\hline \multirow{6}{*}{ OT } & A & 99.98 & 0.31 & 1.27 & 1.64 & 2.90 & $1.94 \pm 0.57$ & 2.90 \\
\hline & B & 114.51 & 0.22 & 0.89 & 1.87 & 4.12 & $2.29 \pm 0.48$ & 3.47 \\
\hline & $\mathrm{C}$ & 162.82 & 0.33 & 1.55 & 1.39 & 3.54 & $2.16 \pm 0.54$ & 3.06 \\
\hline & D & 211.58 & 0.41 & 1.70 & 1.66 & 3.13 & $2.17 \pm 0.76$ & 4.25 \\
\hline & HANDLE & 410.88 & 0.57 & 1.57 & 1.70 & 3.97 & $2.41 \pm 0.63$ & 3.50 \\
\hline & Average & 199.95 & 0.37 & 1.39 & 1.65 & 3.53 & $2.19 \pm 0.59$ & 3.44 \\
\hline \multirow{6}{*}{ EM } & A & 94.07 & 0.79 & 3.69 & 11.02 & 3.49 & $6.07 \pm 2.69$ & 11.89 \\
\hline & B & 110.32 & 0.73 & 4.06 & 9.99 & 4.22 & $6.09 \pm 2.78$ & 12.14 \\
\hline & $\mathrm{C}$ & 160.63 & 0.49 & 4.86 & 10.68 & 3.21 & $6.25 \pm 2.81$ & 12.25 \\
\hline & D & 210.90 & 0.38 & 4.72 & 11.00 & 2.86 & $6.25 \pm 2.77$ & 12.29 \\
\hline & TIP & 8.10 & 1.00 & 4.67 & 9.01 & 2.76 & $5.48 \pm 2.49$ & 11.82 \\
\hline & Average & 116.80 & 0.68 & 4.40 & 10.34 & 3.31 & $6.03 \pm 2.71$ & 12.08 \\
\hline
\end{tabular}

the 28 divot positions, and the trajectories were recorded for a total of 25 seconds for each position, of which 400 samples were used as data for the analysis to average the jitter errors of the tracking signal. TRE was calculated as the RMS difference of the measured divot positions and the tracked tip positions. The inaccuracy of the instrument is defined as the RMS of the errors of all three axes of the systems. Experiment 2 was performed to compare tracking accuracy in a similar scenario with laparoscopic tools. The optical marker plate was located at a distance of approximately $400 \mathrm{~mm}$, which approximately represents the average distance from the tip to the handle of a laparoscopic instrument. Whereas, the EMT sensor was attached close to the tip of the tool.

The aim of this study was to test the hypothesis that there is a difference between TRE for EMT and OT tracked tools at multiple distances from the target tip. The two separate experimental results were analyzed using IBM SPSS software ${ }^{\circledR}[16]$.

\section{RESULTS}

For Experiment 1, a factorial ANOVA i.e. two-way analysis of variance, was conducted to compare the main effects of navigation technology and distance from target position and the effect of interaction between them on the accuracy. Levene's test of equality of variances deemed significant results, therefore a p-value of 0.001 was used to test significance of the results.

The main effect for tracking technology yielded significant results, with a $F$ ratio of $F(1,204)=201.596$ and $p=2.8377 \mathrm{E}-32$ $(\mathrm{p}<0.05)$, indicating a significant difference between accuracy in millimeters of OT $(\mu=2.14, \sigma 2=0.61, n=104)$ and EMT $(\mu=6.15, \sigma 2=2.78, n=108)$. This is also visible in the results provided in Table 1. The main effect for position did not deem significant results, $F(3,204)=0.113$ with $p=0.952(p>0.05)$ while comparing the 4 different distances tested for TRE. No significant interaction effects were found between the two factors.
With respects to Experiment 2, an independent samples Ttest analysis of the data was performed to evaluate the TRE in both EMT and OT sensors with the EMT sensor at the tip and the OT at the length of a laparoscopic instrument. Levene's test for equality of variance revealed unequal variance between the two conditions $\mathrm{F}(2.161)=4.6 \mathrm{E}-5$, hence equal variances were not assumed for this experiment. Significant differences were found in the scores for EMT $(\mu=2.41, \sigma 2=0.64, \mathrm{n}=27)$ and OT $(\mu=5.48, \sigma 2=2.54, \mathrm{n}=27) ; \mathrm{t}(29.439)=-6.081$ with $\mathrm{p}=1.2 \mathrm{E}-6 \quad(\mathrm{p}<0.05)$. This reflects the results found for Experiment 1.

\section{DISCUSSION}

Based on the study conducted in this paper, it can be inferred that OT performs more accurately than EMT, in terms of tracking the tip of the instrument. This was tested in several target positions on different orientation planes, throughout a precisely measured verification phantom. OT was found to be more accurate in all positions and orientations with respect to EMT. However, during the experimental tests, it was found that one of the positions of the phantom was not possible to track using optical tracking due to lack of line of sight between that position and the Polaris cameras, whereas this was not a problem for tracking using the EMT sensor.

OT showed reliable tracking information throughout all positions of the phantom, with an average TRE over the axes and distances from tip of $2.19 \mathrm{~mm}$. This means that if the markers are placed approximately at a distance of $200 \mathrm{~mm}$ from the tip, the accuracy of optical tracking at the tip will be approximately around $2 \mathrm{~mm}$, as reported in Table 1 . The error is larger in the $z$ axis of the Polaris ${ }^{\circledR}$ camera. This is due to error in triangulation of the light rays in depth of the camera and has been verified in multiple studies on OT accuracy [10].

The inaccuracy of EMT, with an average TRE of $6.03 \mathrm{~mm}$, is dependent on several factors, which include: inaccuracy of the pivoting calibration to the tip of the instrument, influence of ferromagnetic equipment such as the surgical table and the tools present in the OR and inhomogeneity of the measurement volume of EMT generator. Pivot calibration was considered to 
be the largest contribution to the increase of error in EMT, as this is dependent both on the type of algorithm used and the sampling frequency of the tracking system. For this research, pivot calibrations were performed through collection in a 20 seconds interval for both systems. Thus, the lower sampling frequency of the Aurora ${ }^{\circledR}$ EMT system $(40 \mathrm{~Hz})$, compared to that of Polaris ${ }^{\circledR}(60 \mathrm{~Hz})$, contributed to a less accurate calibration. The effect of interference from metallic instrumentation, including the surgical table, has been researched throughout several studies [7], [8], [14], [17] and calibration of the distortion is an ongoing topic to reduce this error [11].

Overall, in order to decrease the TRE for EMT tracked instrument tip positions, the suggestion is to position the EMT sensor as close as possible to the tracked position of interest. However pivot calibration for EMT tracked instruments should also be avoided to determine the offset relative to the tip since it can cause a larger error in OR settings: at $8 \mathrm{~mm}$ distance offset from the tool (position TIP in Table 1), the mean error was found to be $5.48 \mathrm{~mm}$. Hence, the best solution could be to make use of surgical equipment with manufactured incorporated EMT sensors and which therefore provide a correct geometry of the tool. From Experiment 2, it is also deducible that OT represents a more accurate and reliable tracking technology, even when, for an EMT system, the sensor is positioned close to the tip (TIP) and the OT markers located at a distance equivalent to that of the handle of a laparoscopic instrument (position HANDLE in Table 1). Further studies should be conducted using TRE assessment for EMT tracked instruments without introduction of inaccuracy of pivoting calibration e.g. using tools with fixed geometries.

\section{CONCLUSIONS}

The presented study aimed to assess the accuracy of OT and EMT systems at multiple distances on surgical tools. This definition of accuracy is known as target registration error. TRE was analyzed through empirical testing using a precisely measured phantom in an OR to study navigation systems for surgical conditions. Results show the outperformance of OT systems compared to EMT. This was found to be valid also in conditions in which an EMT sensor is placed close to the tip of the surgical tool and the OT marker is clamped to the handle of the instrument. The experiments also showed the advantage of EMT of robustness to occlusions, which allowed this tracking system to sample a point which instead could not be tracked by the OT system.

Therefore, EMT technology is of great interest in laparoscopic environment to track flexible instruments. However, for rigid instruments such as a laparoscope camera or a grasper, OT systems provide greater accuracy at all distances along the instrument. The reported accuracy for EMT sensors is approximately $1 \mathrm{~mm}$. However, this is only a measure of how accurate the sensor itself is, similarly to FLE [9], and not how accurately other parts of the instrument can be tracked based on the position of a sensor attached to the instrument. Therefore, further studies should be conducted to better assess TRE for EMT tools under multiple ferromagnetic influences e.g., C-arms, but also with multiple experimental conditions, e.g. using NDI Tabletop Field Generator ${ }^{\circledR}$. These results should also be compared to those of FLE for EMT sensors, similarly to what had been studied in [7], [8], [14], [17].

\section{ACKNOWLEDGMENTS AND DECLARATIONS}

The authors of this study would like to express their gratitude to all the people that contributed in this research. Especially the researchers of the Medical Research group, SINTEF Technology and Society, Trondheim, and The Intervention Centre, Rikshospitalet, Oslo. Special thanks to medical technician Stig Tore Svee, from the Department of Medico-technical Equipment, St. Olav's hospital, Trondheim.

No experimental procedures were conducted in human or animal subjects for the study presented in this paper. No potential conflict of interest was also reported by the authors.

\section{REFERENCES}

[1] C. F. Freyschlag et al., "Optical Neuronavigation without Rigid Head Fixation During Awake Surgery," World Neurosurg., vol. 97, pp. 669-673, 2017.

[2] C. Winne, M. Khan, F. Stopp, E. Jank, and E. Keeve, "Overlay visualization in endoscopic ENT surgery," Int. J. Comput. Assist. Radiol. Surg., vol. 6, no. 3, pp. 401-406, 2011.

[3] P. Mountney, J. Fallert, S. Nicolau, L. Soler, and P. W. Mewes, "An Augmented Reality Framework for Soft Tissue Surgery," Med. Image Comput. Comput. Interv. -- MICCAI 2014, vol. 8673, pp. 423-431, 2014.

[4] L. Bond, B. Schulz, T. VanMeter, and R. C. G. Martin, "Intraoperative navigation of a 3-dimensional needle localization system for precision of irreversible electroporation needles in locally advanced pancreatic cancer," Eur. J. Surg. Oncol., vol. 43, no. 2, pp. 337-343, 2017.

[5] M. Feuerstein, T. Mussack, S. M. Heining, and N. Navab, "Intraoperative laparoscope augmentation for port placement and resection planning in minimally invasive liver resection," IEEE Trans. Med. Imaging, vol. 27, no. 3, pp. 355-369, 2008.

[6] F. X. Bosch, J. Ribes, M. Díaz, and R. Cléries, "Primary liver cancer: Worldwide incidence and trends," Gastroenterology, vol. 127, no. SUPPL., pp. 5-16, 2004.

[7] L. Maier-Hein et al., "Standardized assessment of new electromagnetic field generators in an interventional radiology setting," Med. Phys., vol. 39, no. 6Part1, pp. 3424-3434, 2012.

[8] L. E. B $\emptyset$, H. O. Leira, G. A. Tangen, E. F. Hofstad, T. Amundsen, and T. Langø, "Accuracy of electromagnetic tracking with a prototype field generator in an interventional OR setting," Med. Phys., vol. 39, no. 1, pp. 399-406, 2011.

[9] J. M. Fitzpatrick, "Fiducial registration error and target registration error are uncorrelated," vol. 7261, p. 726102, 2009.

[10] R. Elfring, M. De La Fuente, and K. Radermacher, “Assessment of optical localizer accuracy for computer aided surgery systems," Comput. Aided Surg., vol. 15, no. 1-3, pp. 1-12, 2010.

[11] A. M. Franz, T. Haidegger, W. Birkfellner, K. Cleary, T. M. Peters, and L. Maier-Hein, "Electromagnetic tracking in medicine -A review of technology, validation, and applications," IEEE Trans. Med. Imaging, vol. 33, no. 8, pp. 1702-1725, 2014.

[12] ASTM, "Measurement of Positional Accuracy of Computer Assisted Surgical Systems 1," Astm, vol. i, pp. 1-11, 2015.

[13] (C) 2018 Northern Digital Inc., "NDI." [Online]. Available: https://www.ndigital.com/.

[14] Z. Yaniv, E. Wilson, D. Lindisch, and K. Cleary, "Electromagnetic tracking in the clinical environment," Med. Phys., vol. 36, no. 3, pp. 876-892, 2009.

[15] Mitutoyo Corporation, "AT115 Linear Scales." p. 1.

[16] "SPSS IMB Statistics 25." [Online]. Available: https://www.ibm.com/products/spss-statistics.

[17] E. Shen, G. Shechter, J. Kruecker, and D. Stanton, “Quantification of AC electromagnetic tracking system accuracy in a CT scanner environment," vol. 6918, no. 914, p. 65090L, 2007. 